\title{
Kawasaki Disease Features and Myocarditis in a Patient with COVID-19
}

\author{
Joanne S. Chiu ${ }^{1,3} \cdot$ Manuella Lahoud-Rahme ${ }^{1,3} \cdot$ David Schaffer $^{2} \cdot$ Ari Cohen $^{1,2} \cdot$ Margaret Samuels-Kalow $^{1,2}$
}

Received: 2 May 2020 / Accepted: 9 June 2020 / Published online: 15 June 2020

(c) Springer Science+Business Media, LLC, part of Springer Nature 2020

\begin{abstract}
A 10-year-old male with prolonged fever, rash, and conjunctivitis presented to the emergency department with concern for Kawasaki disease, found to have myocarditis and PCR positive for SARS-CoV-2.
\end{abstract}

Keywords Kawasaki $\cdot$ COVID-19 $\cdot$ Myocarditis

\section{Introduction}

Despite the increasing prevalence and disease burden of severe acute respiratory syndrome coronavirus 2 (SARS$\mathrm{CoV}-2$ ), there is limited understanding of the spectrum of pediatric presentations. Although prior reports have reported that over $90 \%$ of pediatric cases are asymptomatic, mild, or moderate [1], it is critically important for physicians to recognize the possibility of severe presentations in pediatric patients.

\section{Narrative}

A 10-year-old healthy male presented with fever for 7 days, fatigue, diarrhea, cough, rash, and conjunctivitis. He had been evaluated 3 days prior with a nasal swab negative for SARS-CoV-2. In the interim period, he developed a dry cough, diarrhea, conjunctivitis and development of the rash.

Electronic supplementary material The online version of this article (https://doi.org/10.1007/s00246-020-02393-0) contains supplementary material, which is available to authorized users.

Joanne S. Chiu

jchiu@mgh.harvard.edu

1 Department of Pediatrics, Massachusetts General Hospital, Boston, MA, USA

2 Department of Emergency Medicine, Massachusetts General Hospital, Boston, MA, USA

3 Division of Cardiology, Department of Pediatrics, Massachusetts General Hospital, 55 Fruit Street, Boston, MA, USA
He was referred to the emergency department (ED) with concern for Kawasaki Disease (KD).

On arrival to the ED, the temperature was $40.2{ }^{\circ} \mathrm{C}$, the pulse 168 beats per minute, the blood pressure 95/61, the respiratory rate 24 breaths per minute and the oxygen saturation $96 \%$. He was well appearing with a physical exam significant for injected limbic-sparing conjunctiva without discharge. His oropharynx was minimally erythematous without exudate or lesions. His mucous membranes were moist with mild cracking of his lips. He had no lymphadenopathy and no evidence of meningismus. His cardiac exam revealed tachycardia with no murmur auscultated. His pulses were strong with capillary refill less than $2 \mathrm{~s}$. His pulmonary exam was normal. He did not have abdominal tenderness nor hepatosplenomegaly with normal bowel sounds. He had a diffuse rash most prominent on his extremities that appeared mildly erythematous with blanching macules and patches involving the trunk as well as palms and soles (Fig. 1a). There was no palmar erythema.

Given his fever and tachycardia, he was given $400 \mathrm{mg}$ of ibuprofen orally. An ambulatory saturation was performed by having him march in place while attached to a pulse oximeter. His heart rate increased to 180 beats per minute and oxygen saturation increased to $98 \%$. He became lightheaded, which resolved with laying back down. An intravenous line was placed to obtain labs and provide $500 \mathrm{cc}$ normal saline bolus. Electrocardiogram demonstrated sinus tachycardia with low voltages (Fig. 1b).

Laboratory results were revealing for the following: elevated white blood cell count of $16.8 \mathrm{~K} / \mu \mathrm{L}$, hemoglobin of $12.4 \mathrm{~g} / \mathrm{dL}$, and platelets of $207 \mathrm{~K} / \mu \mathrm{L}$ with a differential with elevated absolute neutrophils of $14.45 \mathrm{~K} / \mu \mathrm{L}$ and depressed absolute lymphocytes of $1.18 \mathrm{~K} / \mu \mathrm{L}$. Basic metabolic panel 


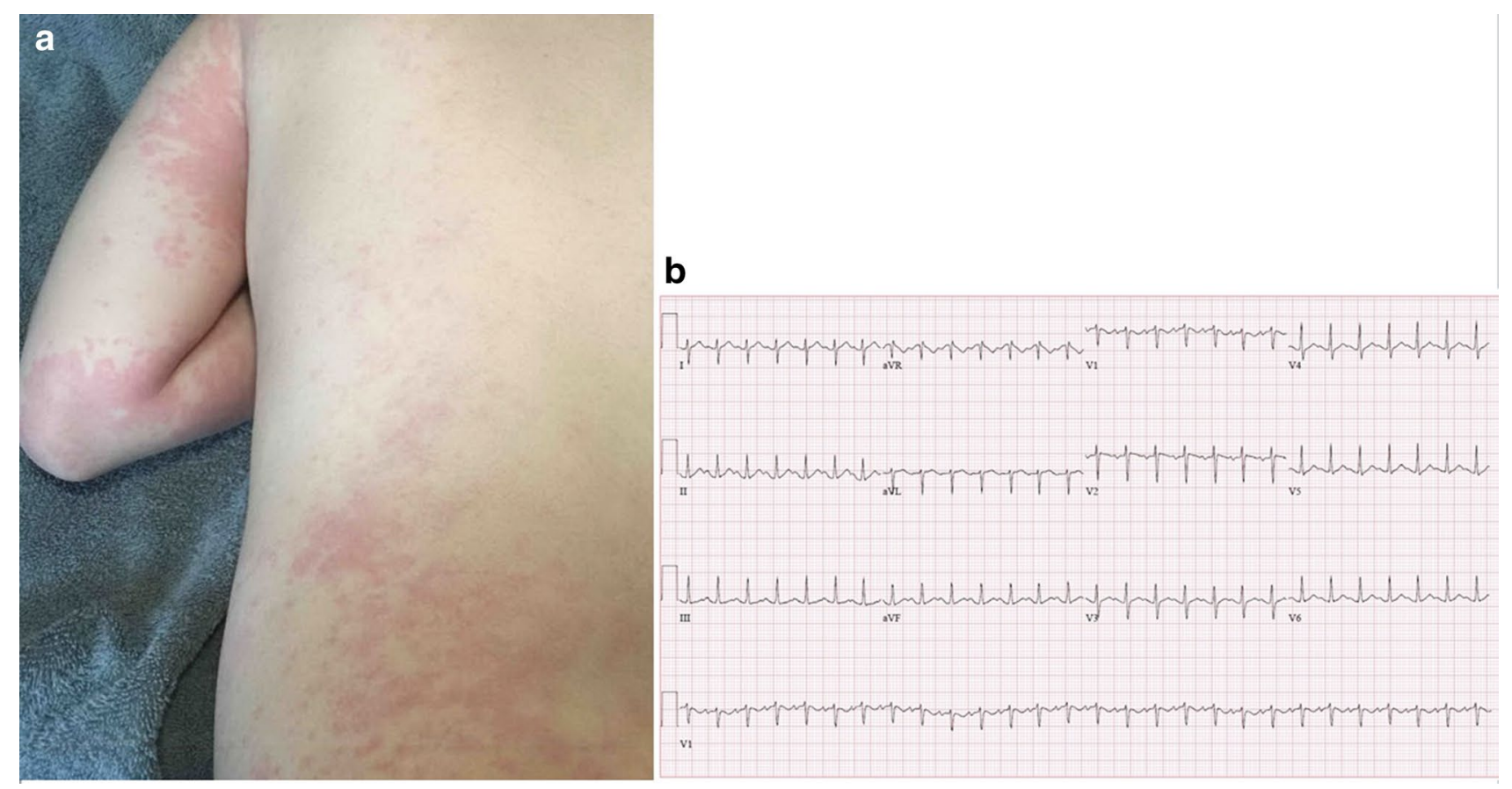

Fig. 1 a Diffuse rash appearing mildly erythematous with blanching macules and patches involving the trunk and extremities. b ECG: Sinus tachycardia with low voltages

was significant for a sodium of $125 \mathrm{mmol} / \mathrm{L}$, potassium of $3.4 \mathrm{mmol} / \mathrm{L}$, chloride $87 \mathrm{mmol} / \mathrm{L}$, bicarbonate of $20 \mathrm{mmol} / \mathrm{L}$ and normal BUN and creatinine. Liver function tests were normal. Phosphorus was low at $2.1 \mathrm{mmol} / \mathrm{L}$. Acute phase reactants were significantly elevated with an erythrocyte sedimentation rate of $57 \mathrm{~mm} / \mathrm{h}$, a c-reactive protein of $280 \mathrm{mg} / \mathrm{L}$, and a procalcitonin of $28 \mathrm{ng} / \mathrm{mL}$ (ref range $0-0.08 \mathrm{ng} / \mathrm{mL}$ ). Specific laboratory tests per our institution-specific COVID-19 algorithm included a D-dimer of $2727 \mathrm{ng} / \mathrm{mL}$, ferritin of $1089 \mu \mathrm{g} / \mathrm{L}$, lactate dehydrogenase of $360 \mathrm{U} / \mathrm{L}$, fibrinogen of $748 \mathrm{mg} / \mathrm{dL}$, and an INR of 1.3 . His creatinine kinase was low at $46 \mathrm{U} / \mathrm{L}$ and high-sensitivity troponin was elevated at $84 \mathrm{ng} / \mathrm{L}$ (ref range $0-14 \mathrm{ng} / \mathrm{L}$ ). His rapid streptococcus test came back negative, EBV serologies were consistent with past infection, and the lactate was normal. His COVID PCR test resulted positive.

With fever reduction and fluid resuscitation, the heart rate decreased to 132 . A venous blood gas was obtained that showed a pH of 7.45, PCO2 of 34 and PO2 of 50 . Bedside ultrasound demonstrated severely diminished left ventricular systolic function with trace pericardial effusion (as shown in the animation Online Resource 1). NT-proBNP assay resulted at $9,477 \mathrm{pg} / \mathrm{mL}$ and the patient became further hypotensive to 75/44. He had diminished peripheral pulses with delayed capillary refill, and a gallop was appreciated upon auscultation. Dopamine was initiated at $5 \mu \mathrm{g} / \mathrm{kg} / \mathrm{min}$ and titrated up to $10 \mu \mathrm{g} / \mathrm{kg} / \mathrm{min}$ with adequate response. Given that the pediatric intensive care unit at our institution had been converted to adult COVID-19 patients, the patient was transferred to an outside institution. At the time of this writing, the patient continues to be critically ill with outcome yet to be determined.

\section{Discussion}

This case illustrates the unique presentation of a COVID19 patient with features of KD and myocarditis, presenting with stable vital signs and a normal ambulatory saturation. To date, there has been one published case of a pediatric COVID-19 patient with concurrent KD without cardiac dysfunction [2] and several case series describing myocarditis as a COVID-19 presentation in the adult population [3-7]. Myocarditis occurs frequently in KD with a prior study demonstrating up to $20 \%$ of patients with left ventricular systolic dysfunction [8]. Additionally, the findings of conjunctivitis and rash have been described as clinical features in the COVID-19 adult population [9], further complicating the interpretation of KD features. Following this experience, our institution has established a pediatric COVID-19 cardiac disease algorithm in the ED that includes initial cardiac biomarkers (high-sensitivity troponin and NT-proBNP) and ECG evaluation for those suspected or confirmed to have COVID-19 who are ill appearing or require admission. Patients presenting with KD features requiring admission now undergo two PCR COVID-19 tests $24 \mathrm{~h}$ apart prior to 
clearance. Further investigation of wide ranging clinical features of COVID-19 in the pediatric population remains urgent.

Funding None.

\section{Compliance with Ethical Standards}

Conflict of Interest The authors declare that they have no conflict of interest.

Ethical Approval This article does not contain any studies with human participants or animals performed by any of the authors.

Informed Consent Not applicable.

\section{References}

1. Dong Y, Mo X, Hu Y et al (2020) Epidemiology of COVID19 among children in China. Pediatrics. https://doi.org/10.1542/ peds.2020-0702

2. Jones VG, Mills M, Suarez D et al (2020) COVID-19 and Kawasaki Disease: novel virus and novel case. Hosp Pediatr 10(6):537-540
3. Lu X, Zhang L, Du H et al (2020) SARS-CoV-2 infection in children. N Engl J Med 382:1663-1665

4. Ruan Q, Yang K, Wang W, Jiang L, Song J (2020) Clinical predictors of mortality due to COVID-19 based on an analysis of data of 150 patients from Wuhan, China. Intensive Care Med 46(5):846-848

5. Zeng JH, Liu YX, Yuan J et al (2020) First case of COVID19 complicated with fulminant myocarditis: a case report and insights. Infection. https://doi.org/10.1007/s15010-020-01424-5

6. Hu H, Ma F, Wei X, Fang Y (2020) Coronavirus fulminant myocarditis saved with glucocorticoid and human immunoglobulin. Eur Heart J. https://doi.org/10.1093/eurheartj/ehaa190

7. Inciardi RM, Lupi L, Zaccone G et al (2020) Cardiac involvement in a patient with Coronavirus Disease 2019 (COVID-19). JAMA Cardiol. https://doi.org/10.1001/jamacardio.2020.1096

8. Printz BF, Sleeper LA, Newburger JW et al (2011) Noncoronary cardiac abnormalities are associated with coronary artery dilation and with laboratory inflammatory markers in acute Kawasaki disease. J Am Coll Cardiol 57:86-92

9. Guan WJ, Ni ZY, Hu Y et al (2020) Clinical characteristics of Coronavirus Disease 2019 in China. N Engl J Med 382(18):1708-1720

Publisher's Note Springer Nature remains neutral with regard to jurisdictional claims in published maps and institutional affiliations. 